\title{
Damping down alcohol dependence
}

Stress is a well-known trigger of alcoholism relapse in susceptible individuals, and it has been suggested that neural systems that mediate behavioural stress responses could be targets for pharmacotherapy of alcoholism. Now, Heilig and colleagues demonstrate that antagonism of neurokinin 1 receptor (NK1R; also known as TACR1) - a receptor that is highly expressed in brain areas involved in stress responses and brain reward - effectively reduces alcohol cravings.

Previous studies have shown that genetic deletion or pharmacological blockade of NK1R dampens behavioural responses to psychological stressors. So, the authors proposed that modulation of NK1R signalling may also influence stress- and rewardrelated processes that are important for excessive alcohol use and relapse.

The hypothesis was first tested in genetically modified mice.

$N k 1 r^{-1-}$ mice that had continuous alcohol availability had markedly lower alcohol consumption levels compared with wild-type mice. Effects were starker when the alcohol concentrations were at the high-end of the concentrations tested (3-15\%), when pharmacological effects override other motivations for alcohol intake, such as taste. Heterozygous mice had similar alcohol consumption levels to controls, indicating that near complete inactivation of NK1R was necessary to suppress alcohol intake.

In light of these results, a placebocontrolled study was then carried out in humans (25 participants per group). A high-affinity NK1R-selective antagonist - LY686017 — that is well-tolerated, orally available and brain penetrant was tested for efficacy in reducing cravings in alcohol-dependent subjects.

As NK1R is important in stress and anxiety responses, subjects with high trait anxiety were targeted for this study. Participants treated with LY686017 demonstrated suppressed spontaneous alcohol cravings as measured by the Alcohol Urge Questionnaire. Furthermore, cravings were also reduced in subjects when they were subjected to a combined stress and alcohol-cue challenge aimed at simulating a real-life situation of high relapse risk. It was also demonstrated that those receiving LY686017 have less activation in the insula - a brain region whose activation correlates with subjective measures of craving - when shown images that produce negative emotional responses.

Overall, the data presented suggest that NK1R antagonism might be of therapeutic value in alcoholism. However, as the authors point out, larger trials, stratified for anxiety measures, will be required to address

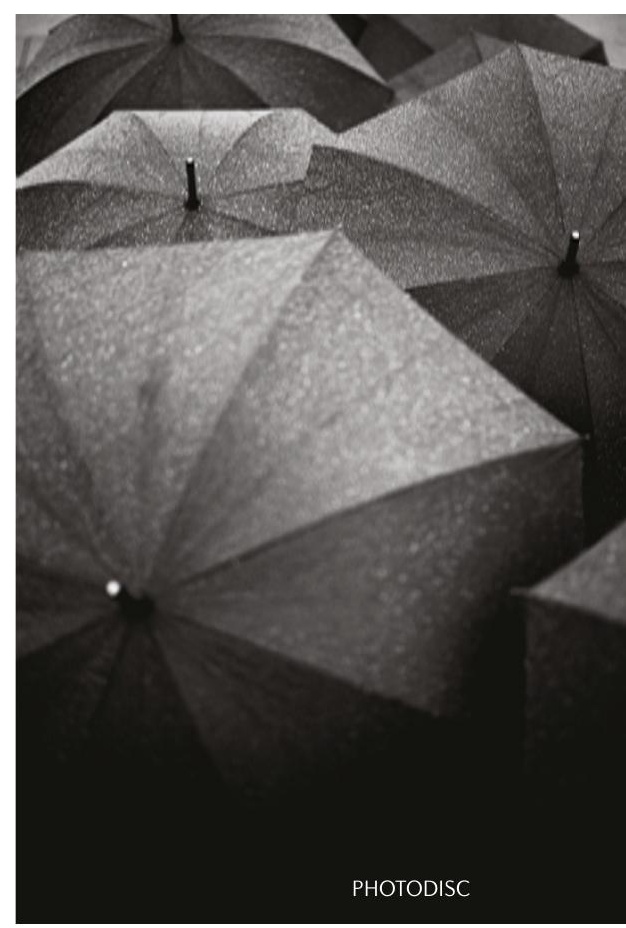

whether the effects of NK1R antagonism are specific for this population.

$$
\begin{array}{r}
\text { Man Tsuey Tse } \\
\text { Copy Editor, } \\
\text { Nature Reviews Drug Discovery }
\end{array}
$$

ORIGINAL RESEARCH PAPER George, T. D. et al. Neurokinin 1 receptor antagonism as a possible therapy for alcoholism. Science 14 Feb 2008 (doi: 10.1126/science.1153813) FURTHER READING Heilig, M. \& Egli, M. Pharmacological treatment of alcohol dependence: target symptoms and target mechanisms. Pharmacol. Ther. 111, 855-876 (2006) 